\title{
Contextuality and the Single-Qubit Stabilizer Subtheory
}

\author{
Piers Lillystone, ${ }^{1}$ Joel J. Wallman, ${ }^{2}$ and Joseph Emerson ${ }^{2,3}$ \\ ${ }^{1}$ Institute for Quantum Computing and Department of Physics and Astronomy, \\ University of Waterloo, Waterloo, Ontario N2L 3G1, Canada \\ ${ }^{2}$ Institute for Quantum Computing and Department of Applied Mathematics, \\ University of Waterloo, Waterloo, Ontario N2L 3G1, Canada \\ ${ }^{3}$ Canadian Institute for Advanced Research, Toronto, Ontario M5G 1Z8, Canada
}

(Dated: May 15, 2018)

\begin{abstract}
Contextuality is a fundamental non-classical property of quantum theory, which has recently been proven to be a key resource for achieving quantum speed-ups in some leading models of quantum computation. However, which of the forms of contextuality, and how much thereof, are required to obtain a speed-up in an arbitrary model of quantum computation remains unclear. In this paper, we show that the relation between contextuality and a compuational advantage is more complicated than previously thought. We achieve this by proving that generalized contextuality is present even within the simplest subset of quantum operations, the so-called single-qubit stabilizer theory, which offers no computational advantage and was previously believed to be completely non-contextual. However, the contextuality of the single-qubit stabilizer theory can be confined to transformations. Therefore our result also demonstrates that the commonly considered prepare-and-measure scenarios (which ignore transformations) do not fully capture the contextuality of quantum theory.
\end{abstract}

Contextuality [1-7], which includes the better-known concept of Bell non-locality as a special case, is often regarded as the fundamental non-classical property of quantum theory. Furthermore, contextuality has emerged as an intriguing explanation for the power of quantum computation: as contextuality is required [8-11] to achieve an exponential quantum speedup by injecting magic states into Clifford circuits [12]; and also quantifies the computational advantage that can be obtained $[10,13-16]$ in both the magic-state and measurement-based models of quantum computation [17].

These considerations motivate us to understand the scope of phenomena that exhibit contextuality, with the aim of identifying which features of contextual phenomena enable quantum computational speed-up. However, one of the primary obstacles to understanding how contextuality powers a quantum computer is that the multi-qubit stabilizer subtheory [18] exhibits contextuality and yet can be efficiently simulated on a classical computer [19, 20].

There are two leading definitions of contextuality: traditional contextuality $[1-5]$ and generalized contextuality $[6,7]$. In this paper, we show that, generalized contextuality [6] is present even in the single-qubit stabilizer subtheory of quantum theory, a fact missed by previous work [21-23]. We further demonstrate that the contextuality present in the single-qubit stabilizer subtheory can be confined to only appear in the transformations. This contradicts the common - and often implicit assumption that an operational theory can be classified as contextual or non-contextual by only considering the preparations and measurements [10, 24-28].

Operational theories and ontological models thereof.An operational theory is non-contextual under a given definition if there exists an ontological model of the operational theory satisfying a specific property that we describe below.

An operational theory is a mathematical framework for predicting the outcomes of an experimental procedure, that is, a sequence of preparations, transformations, and measurements. These elements fully determine the experimental statistics, that is, the conditional probabilities $\operatorname{Pr}(k \mid P, T, M)$ of observing the outcome $k$ when the preparation $P$, transformation $T$, and measurement $M$ are performed sequentially. In quantum mechanics, the conditional probabilities for an experiment consisting of preparing a density matrix $\rho$, applying a completely positive and trace-preserving (CPTP) map $\Phi$, and measuring a positive-operator-valued measure (POVM) $\left\{E_{k}\right\}$ are $\operatorname{Pr}\left(k \mid \rho, \Phi, E_{k}\right)=\operatorname{Tr}\left[\mathrm{E}_{\mathrm{k}} \Phi(\rho)\right]$ by the Born rule.

We can describe the underlying physical processes that generate the experimental statistics using the ontological models formalism. Here we follow the treatment in Ref. [29]. An ontological model is defined by a measurable space $\Lambda$ of possible physical states, with an associated $\sigma$-algebra $\Sigma$, and sets of measures or measurable functions over $\Lambda$ are used to represent preparations, transformations and measurements in the ontological model. For simplicity, we assume that there exists a measure that dominates all other measures in the model [30] (see the Appendix for a proof of the main theorem without this assumption). This allows us to express an ontological model in terms of probability densities, stochastic matrices, and response functions.

When a system is prepared via some procedure $P$, the physical properties of the system are probabilistically assigned values, which are completely encoded by the physical states $\lambda \in \Lambda$. Mathematically, we associate each preparation procedure $P$ with a probability density over 
$\Lambda, \mu_{P}: \Lambda \rightarrow[0,1]$, where $\int_{\Lambda} \mu_{P}(\lambda) d \lambda=1$ as a system is always in some physical state. That is, the probability that a physical state, $\lambda \in \Lambda$, was prepared via $P$ is $\mu_{P}(\lambda)$.

Similarly, when a transformation is applied to a system, the physical properties of the system dynamically evolve according to some stochastic map. Formally, we associate each transformation procedure $T$ with a stochastic map $\Gamma_{T}: \Lambda \times \Lambda \rightarrow[0,1]$, where the conditional probability that some $\lambda$ is sent to another $\lambda^{\prime}$ by $T$ is $\Gamma_{T}\left(\lambda^{\prime}, \lambda\right)$. As every physical state is mapped to some physical state by a transformation, $\int_{\Lambda} \Gamma_{T}\left(\lambda^{\prime}, \lambda\right) d \lambda^{\prime}=1$ for all $\lambda \in \Lambda$.

Finally, when a system is measured via some procedure $M$, the probability that outcome $k$ occurs is specified by the physical state. That is, a measurement $M$ is equivalent to a set of conditional probability functions $\left\{\xi_{k}^{M}: \Lambda \rightarrow[0,1]\right\}_{k}$. As some measurement outcome always occurs, $\sum_{k} \xi_{k}^{M}(\lambda)=1$ for all $\lambda \in \Lambda$. To correctly reproduce the experimental statistics of the operational theory, the distributions must satisfy

$$
\operatorname{Pr}(k \mid P, T, M)=\int_{\Lambda} \xi_{k}^{M}\left(\lambda^{\prime}\right) \Gamma_{T}\left(\lambda^{\prime}, \lambda\right) \mu_{P}(\lambda) d \lambda d \lambda^{\prime} .
$$

Ontological models are assumed, often implicitly [31], to be convex-linear, that is, a probabilistic implementation of a set of operations is represented by the probabilistic mixture of the corresponding probability densities.

Generalized Contextuality.-We now review generalized contextuality. The (experimental) setting of an operation is the set of other operations that are performed with the operation during an experiment. Two operations are operationally equivalent, denoted $\cong$, if they produce the same outcome statistics in all settings.

- Two preparations $P$ and $P^{\prime}$ are equivalent, $(P \cong$ $\left.P^{\prime}\right)$, if $\operatorname{Pr}(k \mid P, T, M)=\operatorname{Pr}\left(k \mid P^{\prime}, T, M\right) \forall T, M$;

- Two transformations $T$ and $T^{\prime}$ are equivalent, $(T \cong$ $\left.T^{\prime}\right)$, if $\operatorname{Pr}(k \mid P, T, M)=\operatorname{Pr}\left(k \mid P, T^{\prime}, M\right) \forall P, M$; and

- Two measurement outcomes $k \in M$ and $k \in M^{\prime}$ are equivalent, $\left[(k, M) \cong\left(k, M^{\prime}\right)\right]$, if $\operatorname{Pr}(k \mid P, T, M)=$ $\operatorname{Pr}\left(k \mid P, T, M^{\prime}\right) \forall P, T$.

Note that the definition of operational equivalence differs slightly from that of Ref. [6] in that we consider operational equivalence of individual measurement outcomes. However, this definition can be obtained from that of Ref. [6] by coarse-graining all measurements into twooutcome POVMs [26, 32, 33].

An ontological model is preparation non-contextual (PNC) if operationally equivalent preparation procedures are represented by the same probability densities, that is,

$$
\mu_{P}=\mu_{P^{\prime}} \Leftrightarrow P \cong P^{\prime} .
$$

Similarly, an ontological model is transformation noncontextual (TNC) if

$$
\Gamma_{T}=\Gamma_{T^{\prime}} \Leftrightarrow T \cong T^{\prime}
$$

and measurement non-contextual (MNC) if

$$
\xi_{k, M}=\xi_{k, M^{\prime}} \Leftrightarrow(k, M) \cong\left(k, M^{\prime}\right) .
$$

An ontological model is universally non-contextual, in the generalized sense, if it satisfies eqs. (2) to (4), otherwise it is contextual [6].

Even a single qubit manifests generalized contextuality [6]. However, previous proofs of generalized contextuality for a single qubit have required subtheories strictly larger the single-qubit stabilizer subtheory.

Contextuality in the 8-state model. - We now show that the 8-state model of the single-qubit stabilizer subtheory exhibits transformation contextuality, a feature missed in previous studies of this model [21-23]. The singlequbit stabilizer subtheory consists of preparations and measurements in the eigenbases of the single-qubit Pauli matrices $\{X, Y, Z\}$, the group of unitary transformations that permute the signed single-qubit Pauli matrices (i.e. the single-qubit Clifford group) and convex combinations of these operations. The single-qubit stabilizer subtheory has the property that preparing an eigenstate of one Pauli matrix $P$ with eigenvalue $\eta$ then measuring another Pauli $Q$ results in the eigenvalue $\eta^{\prime}= \pm \eta$ if $P= \pm Q$ and otherwise results in either eigenvalue with equal probability.

The 8-state model, originally developed in Ref. [21], is a natural ontological model for the single-qubit stabilizer subtheory (see fig. 1). It is defined by setting $\Lambda=\{ \pm 1\}^{\times 3}$ and writing $\lambda=(x, y, z)$, where $x, y$, and $z$ are the eigenvalues of $X, Y$, and $Z$ respectively. These ontic states form the extremal points of the classical probability simplex for three random binary variables. Preparing the $\eta$ eigenstate of $X$ corresponds to setting $x=\eta$ and choosing $y$ and $z$ uniformly at random, etc. Similarly, measuring $X$ returns the value of $x$, etc. This model is both preparation and measurement non-contextual [21].

In the 8-state model, a transformation corresponds to a permutation that acts on the hidden variable $(x, y, z)$ in the same way that it acts on the Pauli operators $(X, Y, Z)$. For example, conjugation by $X$ maps $(X, Y, Z) \rightarrow(X,-Y,-Z)$, and so is represented by the permutation $\Gamma_{X}:(x, y, z) \rightarrow(x,-y,-z)$, with the transformations for $Y$ and $Z$ defined in a similar manner. Conjugation by the Hadamard matrix,

$$
H=\frac{1}{\sqrt{2}}\left(\begin{array}{cc}
1 & 1 \\
1 & -1
\end{array}\right)
$$

maps $(X, Y, Z) \rightarrow(Z,-Y, X)$ and so is represented by the permutation $\Gamma_{H}:(x, y, z) \rightarrow(z,-y, x)$. Note that a Pauli operation preserves the parity $x y z$ and the Hadamard swaps it (as does the phase gate $P$ ). 


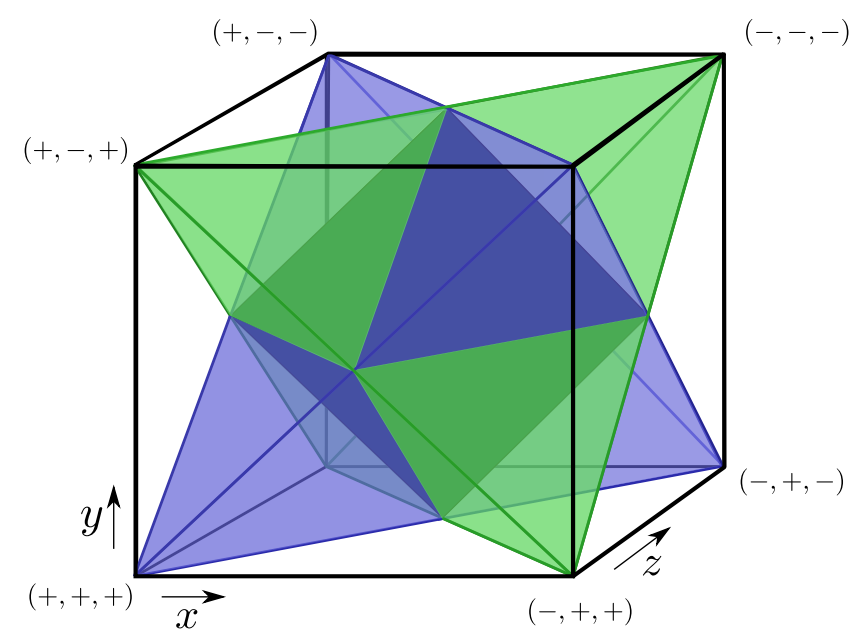

FIG. 1. A graphical representation of the ontic space $\Lambda$ of the 8-state model, where the tuples $(x, y, z)$ index ontic states. The green and blue tetrahedra are the simplices of the oddand even-parity ontic states respectively. The stabilizer polytope is the octahedron defined by the intersection of the two tetrahedra. The transformation $\Gamma_{\mathcal{T}_{1}}$ from eq. (6) maps any ontic state in one of the tetrahedra to another ontic state in the same tetrahedra, while the operationally equivalent transformation $\Gamma_{\mathcal{T}_{2}}$ from eq. (7) maps any ontic state to an ontic state in the opposite tetrahedra.

We now show the 8-state model is transformation contextual. Let

$$
\begin{aligned}
\mathcal{T}_{1}(\rho) & =[\rho+X \rho X+Y \rho Y+Z \rho Z] / 4 \\
, \mathcal{T}_{2}(\rho) & =H \mathcal{T}_{1}(\rho) H .
\end{aligned}
$$

These two transformations are operationally equivalent, as $\mathcal{T}_{1}(\rho)=\mathcal{T}_{2}(\rho)=I / 2$ for any input state $\rho$. However, by convexity we have

$$
\Gamma_{\mathcal{T}_{1}}[(a, b, c),(x, y, z)]= \begin{cases}\frac{1}{4} & \text { if } x y z=a b c \\ 0 & \text { otherwise }\end{cases}
$$

while, since the Hadamard swaps the sign of $x y z$,

$$
\Gamma_{\mathcal{T}_{2}}[(a, b, c),(x, y, z)]= \begin{cases}0 & \text { if } x y z=a b c \\ \frac{1}{4} & \text { otherwise }\end{cases}
$$

That is, $\Gamma_{\mathcal{T}_{1}} \neq \Gamma_{\mathcal{T}_{2}}$, as illustrated in fig. 1 .

The single-qubit stabilizer subtheoy is contextual. Above we demonstrated that the 8-state model for the single-qubit stabilizer subtheory is transformation contextual. We now prove that there is no generalized noncontextual model for the single-qubit stabilizer subtheory, and hence that the single-qubit stabilizer subtheory is contextual. The proof follows by reducing the ontic space of a general preparation non-contextual model of the single-qubit stabilizer subtheory to that of the 8-state model.
Theorem 1. Every ontological model of the single-qubit stabilizer subtheory is either preparation or transformation contextual.

Proof. Fix an arbitrary preparation non-contextual ontological model of the single-qubit stabilizer subtheory. Let $\Delta_{\rho}$ be the support of the quantum state $\rho$ in the ontological model, that is the set of physical states $\rho$ has some possibility of preparing,

$$
\Delta_{\rho}=\left\{\lambda \mid \mu_{\rho}(\lambda)>0, \lambda \in \Lambda\right\} .
$$

Deleting any ontic state $\lambda \in \Lambda$ such that $\mu_{I / 2}(\lambda)=0$, we can partition $\Lambda$ into 8 disjoint spanning sets from the assumption of PNC [6, eqs. (11) and (83)-(87)],

$$
\Lambda_{x, y, z}=\Delta_{(I+x X) / 2} \cap \Delta_{(I+y Y) / 2} \cap \Delta_{(I+z Z) / 2} .
$$

As the model is preparation non-contextual, every quantum state has a unique support. Hence this partitioning is unique.

Noting that preparing $\sigma$ and then applying a transformation $T$, that implements a CPTP map $\Phi$, is a valid preparation procedure for the state $\Phi(\sigma)$. It must be the case that $T$ maps the support of $\rho$ to the support of $\Phi(\rho)$ in a preparation non-contextual ontological model;

$$
\Gamma_{T}: \Delta_{\rho} \rightarrow \Delta_{\Phi(\rho)} .
$$

Therefore a Pauli $X$ unitary must be represented by the permutation $\tau_{X}: \Lambda_{x, y, z} \rightarrow \Lambda_{x,-y,-z}$ on the partition $\left\{\Lambda_{x, y, z}\right\}$. Similarly, Pauli $I, Y$, and $Z$ transformations must be represented by the respective permutations $\tau_{I}: \Lambda_{x, y, z} \rightarrow \Lambda_{x, y, z}, \tau_{Y}: \Lambda_{x, y, z} \rightarrow \Lambda_{-x, y,-z}$, and $\tau_{Z}: \Lambda_{x, y, z} \rightarrow \Lambda_{-x,-y, z}$. Therefore by convex linearity there exists an implementation of $\mathcal{T}_{1}$ that has the same stochastic map as eq. (6), when defined over the coarsegrained sets $\Lambda_{x, y, z}$.

Similarly for the Hadamard gate we have the map $\tau_{H}: \Lambda_{x, y, z} \rightarrow \Lambda_{x,-y, z}$. Therefore there exists an implementation of $\mathcal{T}_{2}$ that has the same stochastic map as eq. (7), when defined over the coarse-grained sets $\Lambda_{x, y, z}$. That is $\mathcal{T}_{1} \cong \mathcal{T}_{2}$ and yet they cannot be represented by the same stochastic map in any preparation non-contextual model.

We now show that any model of the single-qubit stabilizer subtheory must be either Kochen-Specker contextual [2] or transformation contextual.

Theorem 2. Every ontological model of the single-qubit stabilizer subtheory is either Kochen-Specker contextual or transformation contextual.

Proof. The proof proceeds in the same manner as theorem 1, where we use the fact that Kochen-Specker 
non-contextuality is implied by the conjunction of outcome determinism and generalized measurement noncontextuality [6]. By outcome determinism, we can partition $\Lambda$ into 8 disjoint sets according to the measurement outcomes;

$$
\widetilde{\Lambda}_{x y z}=\left\{\lambda \mid \xi_{x}^{X}(\lambda)=1, \xi_{y}^{Y}(\lambda)=1, \xi_{z}^{Z}(\lambda)=1\right\},
$$

By measurement non-contextuality, this partitioning is unique. Using the equivalent to eq. (10) for measurements, the maps $\Gamma_{\mathcal{T}_{1}}$ and $\Gamma_{\mathcal{T}_{2}}$ must be represented as stated in eqs. (6) and (7).

Note theorem 2 can be used to prove theorem 1 , as preparation non-contextuality implies traditional contextuality for sharp measurements [34].

Discussion. - In this Letter we have shown that the single-qubit stabilizer subtheory, a very simple subtheory of the smallest quantum system, exhibits generalized contextuality. This demonstrates that generalized contextuality is so prevalent that even an essentially trivial quantum subtheory is classified as contextual, and therefore non-classical. This contextuality is only apparent if all single-qubit stabilizer operations are accounted for, that is all stabilizer states, all stabilizer measurements, and the full Clifford group. Therefore a universally noncontextual model can only be constructed for strict subtheories of the singe-qubit stabilizer subtheory. For example, the Hadamard and Phase gates are not elements of the toy theory [35] or the standard Wigner function [36], conversely the Hadamard gate is an element of the rebit subtheory [9], but $Y$ eigenstates and $Y$ measurements are not.

Our result also demonstrates that the operational reduction of only considering preparations and measurements is less robust than previously recognized. As this reduction can conceal key features of the model, such as the presence of some forms of contextuality. It is an interesting open problem to understand how and when this kind of reduction can obscure such important conceptual features of an operational theory. A possible route to investigate this is the Choi-Jamiokowski isomorphism. The isomorphism, in quantum theory, relates transformations to states in a larger Hilbert space. Hence by using a similar isomorphism for ontological models we may be able to find a connection between the impossibility of a universal non-contextual ontological model of the single qubit stabilizer subtheory and the impossibility of a preparation non-contextual ontological model of the two-qubit stabilizer subtheory $[5,34]$.

We would like to thank Rob Spekkens, Stephen Bartlett, Angela Karanjai, and Hammam Qassim for useful discussions. This research was supported by the Government of Canada through CFREF Transformative Quantum Technologies program, NSERC Discovery program, and Industry Canada.
[1] J. S. Bell, Rev. Mod. Phys. 38, 447 (1966).

[2] S. Kochen and E. P. Specker, Journal of Mathematics and Mechanics 17, 59 (1967).

[3] N. D. Mermin, Physical Review Letters 65, 3373 (1990).

[4] A. Peres, Journal of Physics A: Mathematical and General 24, L175 (1991).

[5] N. D. Mermin, Reviews of Modern Physics 65, 803 (1993).

[6] R. W. Spekkens, Physical Review A 71, 052108 (2005).

[7] A. W. Simmons, J. J. Wallman, H. Pashayan, S. D. Bartlett, and T. Rudolph, New Journal of Physics 19, 033030 (2017).

[8] M. Howard, J. J. Wallman, V. Veitch, and J. Emerson, Nature 509, 351 (2014).

[9] N. Delfosse, P. Allard Guerin, J. Bian, and R. Raussendorf, Phys. Rev. X 5, 021003 (2015).

[10] J. Bermejo-Vega, N. Delfosse, D. E. Browne, C. Okay, and R. Raussendorf, Phys. Rev. Lett. 119, 120505 (2017).

[11] R. Raussendorf, D. E. Browne, N. Delfosse, C. Okay, and J. Bermejo-Vega, Phys. Rev. A 95, 052334 (2017).

[12] S. Bravyi and A. Kitaev, Phys. Rev. A 71, 022316 (2005).

[13] V. Veitch, C. Ferrie, D. Gross, and J. Emerson, New Journal of Physics 14, 113011 (2012).

[14] V. Veitch, S. A. H. Mousavian, D. Gottesman, and J. Emerson, New Journal of Physics 16, 013009 (2014).

[15] C. Okay, S. Roberts, S. D. Bartlett, and R. Raussendorf, Quantum Information and Computation 17, 1135 (2017).

[16] L. Catani and D. E. Browne, (2017), arXiv:1711.08676v1.

[17] R. Raussendorf and H. J. Briegel, Phys. Rev. Lett. 86, 5188 (2001).

[18] A subtheory of quantum theory is a closed subset of the operations available within quantum theory for a given Hilbert space dimension.

[19] D. Gottesman, Proceedings of the XXII International Colloquium on Group Theoretical Methods in Physics 1, 32 (1998).

[20] S. Aaronson and D. Gottesman, Phys. Rev. A 70, 052328 (2004).

[21] J. J. Wallman and S. D. Bartlett, Physical Review A 85, 062121 (2012).

[22] P. Blasiak, Physics Letters A 377, 847 (2013).

[23] L. Kocia and P. Love, (2017), arXiv:1705.08869v2.

[24] N. Harrigan and T. Rudolph, (2007), arXiv:0709.4266v1.

[25] R. W. Spekkens, Phys. Rev. Lett. 101, 020401 (2008).

[26] R. Kunjwal and R. W. Spekkens, Phys. Rev. Lett. 115, 110403 (2015).

[27] A. Krishna, R. W. Spekkens, and E. Wolfe, (2017), arXiv:1704.01153v2.

[28] D. Schmid, R. W. Spekkens, and E. Wolfe, (2017), arXiv: $1710.08434 \mathrm{v} 1$.

[29] M. S. Leifer, Quanta 3, 67 (2014), arXiv:1409.1570.

[30] That is $\exists \sigma$ such that $\sigma(\Delta)=0 \Rightarrow \mu(\Delta)=0, \Delta \in \Sigma$ for all $\mu$ in the model.

[31] A. Blass and Y. Gurevich, (2015), arXiv:1503.08084.

[32] M. S. Leifer and R. W. Spekkens, International Journal of Theoretical Physics 44, 1977 (2005).

[33] R. W. Spekkens, Foundations of Physics 44, 1125 (2014).

[34] M. S. Leifer and O. J. E. Maroney, Phys. Rev. Lett. 110, 120401 (2013).

[35] R. W. Spekkens, Phys. Rev. A 75, 032110 (2007). 
[36] K. S. Gibbons, M. J. Hoffman, and W. K. Wootters, Phys. Rev. A 70, 062101 (2004).

\section{Appendix: Measure-Theoretic Treatment of Theorem 1}

We now prove theorem 1 in the more general measuretheoretic framework for ontological models, see [29] definition 8.2 .

Theorem 3. Every ontological model of the single-qubit stabilizer subtheory is either preparation or transformation contextual.

Proof. To adapt the proof to the measure-theoretic set- ting, we need to change the definition of support of a quantum state as follows. Let $\Delta_{\rho}$ be the support of the quantum state $\rho$ in the ontological model, that is, a (not necessarily unique) set such that for all $S \in \Sigma$,

$$
\mu_{\rho}(S) \begin{cases}=1 & \text { if } \Delta_{\rho} \subseteq S \\ <1 & \text { otherwise }\end{cases}
$$

As before we delete any measurable set $S \in \Sigma$ such that $\mu_{I / 2}(S)=0$, then partition $\Lambda$ into 8 spanning sets that intersect on sets of measure zero, from the assumption of PNC [6, eqs. (11) and (83)-(87)]. Having reduced the model to a model over a finite set of states, the rest of the proof follows as described in the main text. 